\title{
Leishmania promastigotes: building a safe niche within macrophages
}

\author{
Neda Moradin and Albert Descoteaux* \\ INRS - Institut Armand-Frappier and Center for Host-Parasite Interactions, Laval, QC, Canada
}

\section{Edited by:}

Stephen M. Beverley, Washington

University in St. Louis, USA

Reviewed by:

Anthony P. Sinai, University of

Kentucky College of Medicine, USA

Jean Celli, NIH, USA

*Correspondence:

Albert Descoteaux, Institut National de la Recherche Scientifique, Institut Armand-Frappier, 531 boulevard des Prairies, Laval, OC H7V 1B7, Canada. e-mail: albert.descoteaux@iaf.inrs.ca
Upon their internalization by macrophages, Leishmania promastigotes inhibit phagolysosome biogenesis. The main factor responsible for this inhibition is the promastigote surface glycolipid lipophosphoglycan (LPG). This glycolipid has a profound impact on the phagosome, causing periphagosomal accumulation of F-actin and disruption of phagosomal lipid microdomains. Functionally, this LPG-mediated inhibition of phagosome maturation is characterized by an impaired assembly of the NADPH oxidase and the exclusion of the vesicular proton-ATPase from phagosomes. In this chapter, we review the current knowledge concerning the nature of the intra-macrophage compartment in which Leishmania donovani promastigotes establish infection. We also describe how LPG enables this parasite to remodel the parasitophorous vacuole.

Keywords: Leishmania, macrophage, phagosome, virulence, lipophosphoglycan
The protozoan Leishmania parasitizes phagocytic cells, causing a spectrum of human diseases ranging from a confined cutaneous lesion to a progressive and potentially fatal visceral infection. Leishmania is endemic in 98 countries where it constitutes a serious health problem (Alvar et al., 2012). This parasite exists under two distinct developmental stages. Promastigote forms, which develop within sand flies, are inoculated into the mammalian host upon the bloodmeal of the vector. They are internalized by phagocytes where they subsequently differentiate into amastigotes. To do so, promastigotes must avoid being killed by the antimicrobial activities of macrophages. Amastigotes are fully adapted to the conditions encountered within macrophage phagolysosomes and are responsible for the various pathologies associated with the infection. No effective and safe vaccines are available, and current treatment is based on chemotherapy, which is difficult to administer, expensive, and becoming ineffective due to the spread of drug resistance. Understanding the nature and the functional properties of the vacuoles in which both stages of the parasite are internalized and develop is an important step towards the development of novel approaches to prevent and treat leishmaniases.

\section{THE PHAGOLYSOSOME AS A REPLICATIVE NICHE FOR AMASTIGOTES}

Early work by Alexander and Vickerman (1975) and Chang and Dwyer (1976) revealed that Leishmania amastigotes multiply in macrophages within compartments that fuse with lysosomes. These seminal discoveries established that in mammals, Leishmania resides and proliferates within phagolysosomal compartments of host macrophages. Subsequent work indicated that amastigotes are resistant to the hydrolytic environment prevailing in phagolysosomes (Lewis and Peters, 1977; Chang and Dwyer, 1978). Amastigotes enter macrophages via a Racl- and Arf6-dependent process, and are found in parasitophorous vacuoles that interact with endosomes and lysosomes and acquire lysosomal features (Chang and Dwyer, 1976; Berman et al., 1979; Antoine et al., 1998; Dermine et al., 2001; Lodge and Descoteaux, 2006). Consistently, vacuoles harboring amastigotes contain numerous lysosomal hydrolases and their membranes are enriched with late endosomal/lysosomal proteins, such as Rab7, LAMP-1, and LAMP-2. The vacuolar $\mathrm{H}^{+}$-ATPase present on amastigotes-harboring vacuoles is responsible for the acidic pH (pH 4.7-5.2) (Antoine et al., 1990, 1998; Vinet et al., 2009). In addition, vacuoles harboring amastigotes display molecules characteristic of the endoplasmic reticulum such as calnexin and the membrane fusion regulator Sec22b (Ndjamen et al., 2010). This observation suggests that amastigotes-harboring vacuoles are hybrid compartments composed of both endoplasmic reticulum and endocytic pathway components.

The fact that amastigotes reside in an acidic environment is consistent with their optimal metabolism (respiration, catabolism of energy substrates and incorporation of precursors into macromolecules) at acidic $\mathrm{pH}$ ( $\mathrm{pH} 4.0$ and 5.5), whereas these activities are optimal at neutral $\mathrm{pH}$ for promastigotes (Mukkada et al., 1985). To avoid exposure to oxidants, amastigotes subvert the generation of reactive oxygen species (ROS) within the parasitophorous vacuole through diverse mechanisms including heme degradation and prevention of the NADPH oxidase complex assembly (Pham et al., 2005; Lodge and Descoteaux, 2006). In the latter case, amastigotes evade the phosphorylation of cytosolic $\mathrm{p} 47^{\text {phox }}$, a key event for the NADPH oxidase activation during phagocytosis (Lodge and Descoteaux, 2006). Interestingly, Leishmania donovani amastigotes disrupt the integrity of lipid microdomains present within the phagosomal membrane, as assessed by the alteration of GM1 distribution and the impairment of flotillin recruitment (Lodge and Descoteaux, 2006). Flotillin is a component of lipid microdomains and is recruited to phagosomes during the maturation process from late endocytic organelles. The mechanisms by which $L$. donovani amastigotes disrupt lipid microdomains and the ensuing consequences 
on pathogenesis are not known and remain to be investigated. Whereas amastigotes from most Leishmania species proliferate in tight individual vacuoles, amastigotes of the L. mexicana complex reside in large communal parasitophorous vacuoles (Real et al., 2008). The molecular basis of parasitophorous vacuole enlargement and the consequences for the intracellular survival of these parasites are poorly understood.

\section{ARRESTED PHAGOSOME MATURATION BY PROMASTIGOTES}

In contrast to amastigotes, promastigotes exist only transiently within mammals. Following their inoculation by sand flies, promastigotes must avoid destruction by the innate immune system of their mammalian hosts in order to differentiate into amastigotes. Hence, promastigotes evade the antimicrobial properties of serum components before being internalized by macrophages. Interestingly, recent studies using an experimental model of natural transmission in mice revealed that a portion of sand fly-transmitted promastigotes may reside transiently within neutrophils before being taken up by dendritic cells and macrophages (Peters and Sacks, 2009). The nature and characteristics of the neutrophil compartments in which promastigotes transit are however unknown.

Serum-opsonized promastigotes enter macrophages predominantly via the complement receptor 3 in a process that mainly depends on the GTPase RhoA (Lodge and Descoteaux, 2005b). Focal exocytosis of host cell membrane originating from endosomes, lysosomes, and the endoplasmic reticulum contributes to the formation of the promastigote-containing phagosomes (Gagnon et al., 2002; Vinet et al., 2009; Forestier et al., 2011). Such supply of membrane from various intracellular compartments may contribute to the formation and the composition of the nascent parasitophorous vacuole.

One mechanism used by promastigotes to evade the microbicidal consequences of phagocytosis is the inhibition of phagolysosome biogenesis (Desjardins and Descoteaux, 1997) (Figure 1). Hence, in contrast to amastigotes, L. donovani, L. major, and L. chagasi promastigotes are internalized in phagosomes that poorly interact with late endosomes and lysosomes and which display a delayed recruitment of LAMP-1, possibly a consequence of an impaired recruitment of Rab7 (Desjardins and Descoteaux, 1997; Scianimanico et al., 1999; Dermine et al., 2000; Späth et al., 2003; Gaur et al., 2009; Rodriguez et al., 2011). This effect of Leishmania promastigotes on phagosome-lysosome fusion is restricted to parasite-containing phagosomes, as the fusion machinery remains operational in infected macrophages (Desjardins and Descoteaux, 1997).

Promastigote-induced phagosome maturation arrest is characterized by a periphagosomal F-actin accumulation (Holm et al., 2001), and by the phagosomal retention of components of the actin polymerization machinery, including Arp2/3, WiskottAldrich Syndrome Protein (WASP), $\alpha$-actinin, Myosin II, and Nck (Lodge and Descoteaux, 2005a). The Rho-family GTPases, Cdc42, Rac1, and RhoA are also present on promastigotes-containing phagosomes (Lodge and Descoteaux, 2005a; Lerm et al., 2006). The significance of this build up of periphagosomal F-actin is unclear, but it may contribute to the inhibition of phagosome maturation, possibly by interfering with the recruitment of signal transducers and vesicles trafficking to the forming phagolysosome (Lodge and Descoteaux, 2005a; Lerm et al., 2006). However, periphagosomal F-actin accumulation is not observed with all Leishmania species. Indeed, F-actin rapidly disassembles from newly formed phagosomes harboring L. amazonensis metacyclic promastigotes (Courret et al., 2002). Whether this is related to the fact that $L$. amazonensis induces and resides in spacious communal vacuoles is a possibility that deserves further investigations.

As is the case for amastigotes, promastigotes disrupt lipid microdomains present in the phagosomal membrane (Dermine et al., 2001), as assessed by the inhibition of flotillin recruitment to phagosomes containing promastigotes (Dermine et al., 2001). These membrane microdomains play key roles in various cellular functions, serving as platforms to recruit and concentrate molecules involved in processes such as membrane fusion, generation of microbicidal effectors, and signal transduction (Simons and Vaz, 2004). Targeting and disruption of these structures in phagosomes may thus represent an efficient way for pathogens to subvert the antimicrobial arsenal of macrophages.

\section{FUNCTIONAL CONSEQUENCES OF PHAGOSOME MATURATION ARREST BY PROMASTIGOTES}

What are the functional consequences of the inhibition of phagosome maturation induced by Leishmania promastigotes? An important microbicidal mechanism of macrophages is the generation of ROS (ROS; superoxide anions, hydrogen peroxide) which is mediated by the nicotinamide adenine dinucleotide phosphate (NADPH) oxidase complex. Assembly of the NADPH oxidase complex requires that cytosolic phosphorylated $\mathrm{p} 47^{\text {phox }}$ and $\mathrm{p} 40 / \mathrm{p} 67^{\text {phox }}$ heterodimers associate to form $\mathrm{p} 47 / \mathrm{p} 67 / \mathrm{p} 40^{\text {phox }}$ heterotrimers prior to their membrane translocation, where they interact with membrane-associated flavocytochrome b558 (Bokoch and Diebold, 2002; Brown et al., 2003). Assembly of this complex at the phagosome membrane allows the generation of high levels of ROS within the phagosome. Disabling the NAPDH oxidase is a virulence strategy used by various pathogens to reduce exposure to oxidants (Vazquez-Torres and Fang, 2001; Allen and McCaffrey, 2007; Harada et al., 2007). In the case of Leishmania, uptake of promastigotes triggers the phosphorylation of $4^{p h o x}$ and the formation of heterocomplexes containing both $\mathrm{p} 47^{\text {phox }}$ and p67 $7^{\text {phox }}$ (Lodge et al., 2006). However, these cytosolic components of the NADPH oxidase complex fail to associate to the promastigote-containing vacuoles. Co-internalization of L. donovani promastigotes and IgGcoated erythrocytes revealed that inhibition of ROS production is restricted to promastigote-containing phagosomes. Thus, phagosome maturation arrest induced by $L$. donovani promastigotes enables these parasites to establish infection in an environment devoid of oxidants, which may be favorable to their survival. Interestingly, recruitment of the NADPH oxidase to the phagosome membrane has been shown to limit the proteolytic activity of the phagosome. A tight control of proteolysis within the phagosome is key to an efficient antigen processing and presentation. As activity of the NADPH oxidase plays an important role in the 


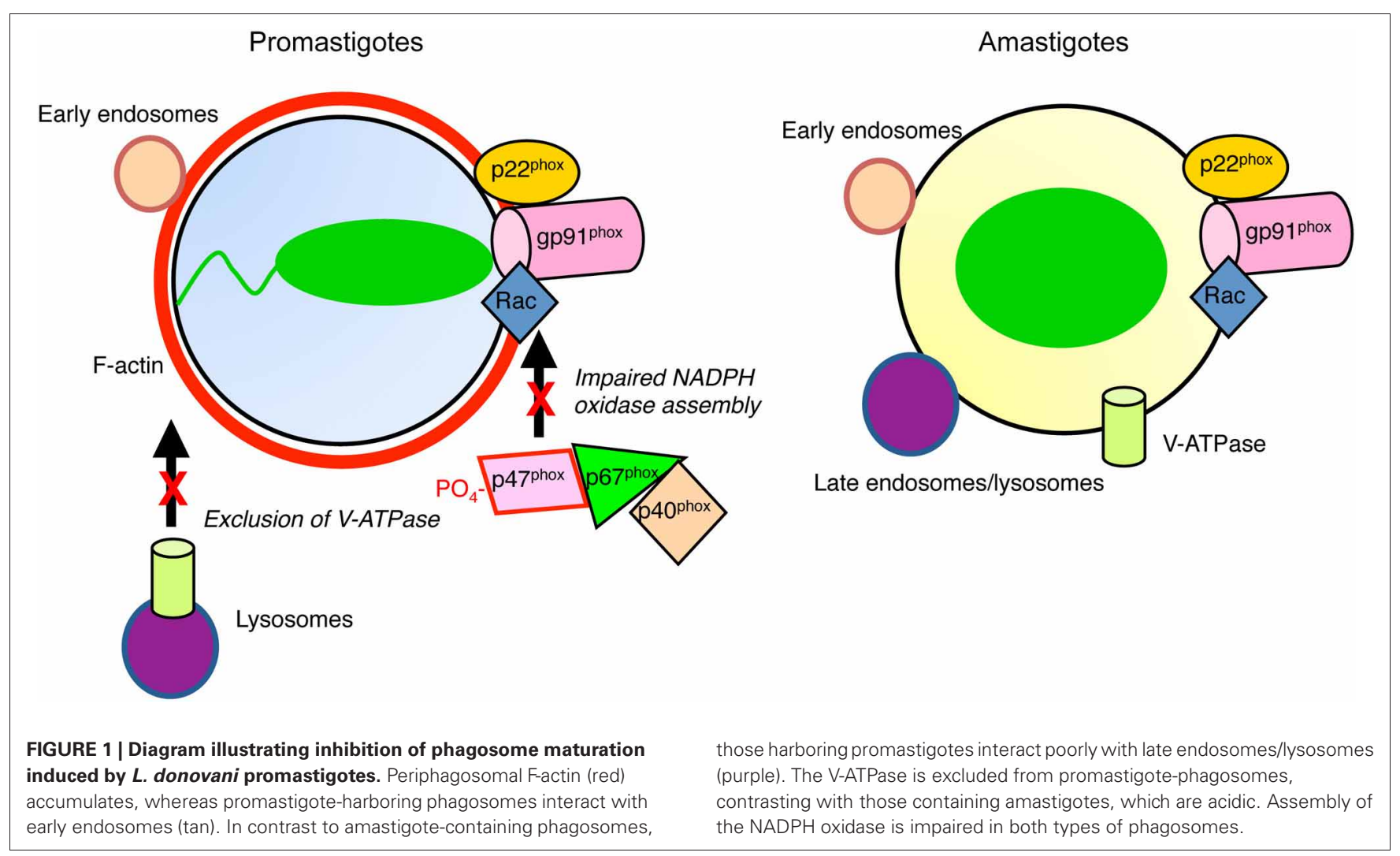

control of antigen presentation (Mantegazza et al., 2008; Savina et al., 2009; Rybicka et al., 2012), its impairment by L. donovani promastigotes may contribute to the evasion of the immune system by this parasite.

Phagosome acidification is essential for the acquisition of microbicidal properties and for optimal antigen processing, as most lysosomal hydrolases are optimally active at acidic $\mathrm{pH}$. Acidification is mediated by the vacuolar proton-ATPase (v-ATPase), which is present on various endocytic organelles (Flannagan et al., 2009). The v-ATPase is a multimeric complex consisting in the multi-subunit cytoplasmic $\mathrm{V}_{1}$-sector that is responsible for ATP hydrolysis, and of the multi-subunit transmembrane $\mathrm{V}_{0}$-sector that pumps protons acrosss the bilayer (Marshansky and Futai, 2008). Several intravacuolar pathogens target this process during establishment of infection. The most notable one is Mycobacterium tuberculosis, which secretes a protein tyrosine phosphatase, PtpA, to prevent recruitment of the v-ATPase to the phagosome (Wong et al., 2011). Similar to phagosomes containing M. tuberculosis, the v-ATPase is excluded from $L$. donovani promastigote-harboring phagosomes, up to $24 \mathrm{~h}$ after phagocytosis (Vinet et al., 2009). This finding provides new insight on our understanding of Leishmania biology. In the absence of data on the $\mathrm{pH}$ of promastigote-containing phagosomes, it has been assumed that promastigotes initiate infection in an acidic environment and that differentiation of promastigotes into amastigotes is mainly triggered by a rapid exposure to an acidic environment and elevated temperature (Zilberstein and Shapira, 1994; Rosenzweig et al., 2008). Exclusion of the v-ATPase suggests that $L$. donovani promastigotes initiate the differentiation process in a non-acidified environment. Further studies will be required to fully address this issue.

\section{PROMASTIGOTE-INDUCED PHAGOSOME REMODELING REOUIRES LIPOPHOSPHOGLYCAN}

An important issue is to understand how Leishmania promastigotes can remodel the vacuoles in which they are internalized. Bacterial pathogens such as Salmonella and Legionella use specialized secretion apparatuses to alter intracellular trafficking and block phagosome maturation (Brumell and Grinstein, 2004; Flannagan et al., 2009). In Leishmania, no such secretion systems have been described so far. However, recent work have revealed that Leishmania promastigotes release microvesicles into the extracellular milieu to deliver cargo into the infected cells (Silverman and Reiner, 2011; Lambertz et al., 2012). These Leishmania exosomes, which contain over 300 proteins, modulate macrophage functions to create an environment permissive for early infection (Silverman et al., 2010; Silverman and Reiner, 2012). The possible involvement of exosomes in promastigoteinduced phagosomes remodeling is an attractive hypothesis that remains to be investigated. This being said, so far, the only Leishmania molecule known to alter intracellular trafficking and to inhibit phagolysosome biogenesis is the abundant surface glycolipid lipophosphoglycan (LPG) (Desjardins and Descoteaux, 1997).

Leishmania synthesizes various glycoconjugates associated to virulence, the most notable belonging to the phosphoglycans family (Descoteaux et al., 1995). These phosphoglycans have in common a unique structure not found in mammals, 
namely the disaccharide-phosphate $\mathrm{Gal}(\beta 1,4) \mathrm{Man}\left(\alpha 1-\mathrm{PO}_{4} \rightarrow 6\right)$ unit (Descoteaux and Turco, 1999). These unique glycoconjugates can be either secreted (phosphoglycan, proteophosphoglycan, and acid phosphatase), or membrane-bound (lipophosphoglycan, also known as LPG). LPG is the most abundant promastigote surface glyconjugate, with 5 million copies per cell, and forms a dense glycocalyx by covering the entire parasite surface. This molecule is promastigote stage-specific, as it is either strongly down-regulated or absent in the amastigote stage (McConville and Blackwell, 1991; Turco and Sacks, 1991). The abundance, location, and uniqueness of these glycoconjugates are consistent with the functions that LPG plays during the establishment of promastigotes within macrophages.

Structurally, LPG consists of a polymer of the repeating $\operatorname{Gal}(\beta 1,4) \mathrm{Man}\left(\alpha 1-\mathrm{PO}_{4} \rightarrow 6\right)$ unit, linked to a 1-O-alkyl-2lyso-phosphatidyl(myo)inositol (PI) anchor via a glycan core (Descoteaux and Turco, 1999). At the non-reducing end of the repeating unit moiety is a small cap composed of neutral oligosaccharides, mostly galactose, and mannose residues. The number of repeating units varies from 16 to 30 per LPG molecule, depending on the promastigote developmental stage (procyclic $v s$ metacyclic) and species. The PI anchor and the glycan core are extensively conserved among Leishmania species (McConville et al., 1990). In contrast, the oligosaccharide cap displays some degree of variability among Leishmania species, in both sugar composition and sequence. The most important differences in the structure of LPG among the various Leishmania species are found within the repeating units. While $L$. donovani LPG follows the basic $\operatorname{Gal}(\beta 1,4) \mathrm{Man}\left(\alpha 1-\mathrm{PO}_{4} \rightarrow 6\right)$ repeat sequence (Thomas et al., 1992), LPG molecules from other species have additional saccharide side chains branching off the $\mathrm{C} 3$ position of the galactose residue.

Using a panoply of $L$. donovani mutants defective in the biosynthesis of LPG, Desjardins and Descoteaux discovered that LPG was the molecule responsible for the inhibition of phagolysosome biogenesis by promastigotes (Desjardins and Descoteaux, 1997). Hence, in contrast to wild type $L$. donovani promastigotes, mutants lacking either LPG or all phosphoglycans were unable alter the fusogenecity of phagosomes toward late endosomes and lysosomes, and did not interfere with the recruitment of the late endocytic and lysosomal markers Rab7 (Scianimanico et al., 1999). These findings are consistent with the observation that amastigotes, which do not make LPG, are internalized into compartments that acquire lysosomal features (Dermine et al., 2000; Courret et al., 2002), including the v-ATPase (Vinet et al., 2009).

Further studies revealed the importance of the length of the LPG repeating unit moiety. Hence, a minimal length appears essential to perturb membrane properties, as was shown in a viral syncytia formation assay and in a PKC membrane association assay (Easterbrook et al., 1995; Giorgione et al., 1996). Consistently, a $L$. donovani mutant expressing truncated forms of LPG with only 3-5 repeating units was unable to inhibit phagosome-endosome fusion (Desjardins and Descoteaux, 1997). In contrast, the level of structural complexity of this moiety, such as the presence of oligosaccharide side-chains, does not affect membrane properties. Indeed, a $L$. major mutant defective in
LPG oligosaccharide side-chain biosynthesis was able to impair phagosome-endosome fusion to the same extent as wild-type L. major promastigotes (Dermine et al., 2000).

Clearly, LPG exerts a profound influence on the composition and properties of the promastigote-harboring phagosomes. From a cell biology stand point, in addition to understanding the biology of Leishmania parasites, this discovery provided a novel and unique system to investigate the process of phagolysosome biogenesis. For both reasons, one important issue was to elucidate the mechanism(s) by which a single microbial-derived glycolipid can affect so profoundly phagosome maturation.

\section{THE IMPACT OF LIPOPHOSPHOGLYCAN ON PHAGOSOME PROPERTIES}

Periphagosomal accumulation of F-actin induced by LPG (Holm et al., 2001) is the consequence of an abnormal retention of the Rho-family GTPase $\mathrm{Cdc} 42$ at the phagosome. This role for $\mathrm{Cdc} 42$ was revealed by expressing the dominant-negative Cdc42N17 mutant in RAW 264.7 macrophages, which inhibited LPG-mediated periphagosomal F-actin accumulation (Lodge and Descoteaux, 2005a; Lerm et al., 2006). Interestingly, the host cell machinery involved in actin polymerization and cytoskeleton rearrangement, such as WASP, Arp2/3, Nck, $\alpha$-actinin, and myosin II, are retained at the phagosome in a LPG-dependent manner, and this can be reversed by the $\mathrm{Cdc} 42 \mathrm{~N} 17$ mutant (Lodge and Descoteaux, 2005a). LPG also interferes with the recruitment of Protein Kinase C (PKC)- $\alpha$ to the phagosome membrane (Holm et al., 2001). PKC- $\alpha$ was shown to participate in periphagosomal $\mathrm{F}$-actin breakdown (Holm et al., 2003) and in the regulation of phagosome maturation (Allen and Aderem, 1995; Aderem and Underhill, 1999; Ng Yan Hing et al., 2004). Whether LPG-mediated exclusion of PKC- $\alpha$ from $L$. donovani promastigote-containing phagosomes contributes to the periphagosomal accumulation of F-actin remains to be demonstrated. Similarly, the consequences of periphagosomal F-actin accumulation on phagosome functions remain to be investigated.

Another mechanism by which LPG exerts its action on phagosome maturation implicates the transfer of LPG from the parasite surface to lipid microdomains present in the phagosome membrane (Tolson et al., 1990). This causes a disorganization of these structures and prevents the formation of new lipid microdomains after phagocytosis. Phagosomal lipid microdomains are central to the recruitment/assembly of the NADPH oxidase and the $\mathrm{v}$-ATPase, and are involved in the regulation of phagosomeendosome fusions (Dermine et al., 2001; Shao et al., 2003; Vilhardt and Van Deurs, 2004). How lipid microdomains regulate interactions between phagosomes and the endocytic system is unclear. However, the observations that proteins involved in membrane fusion are located in lipid microdomains are consistent with these structures acting as fusion sites (Gil et al., 2005; Kay et al., 2006). Insertion of LPG into lipid microdomains via its GPI anchor allows the negatively charged $\mathrm{Gal}(\beta 1,4)$ Man $\left(\alpha 1-\mathrm{PO}_{4} \rightarrow 6\right)$ polymer to directly interfere with the clusterization of molecules into these microdomains (Dermine et al., 2005; Winberg et al., 2009). One direct consequence of LPG-mediated 
microdomain disorganization is the exclusion of the membrane fusion regulator Synaptotagmin (Syt) V from the phagosome (Vinet et al., 2009). Syt V plays a regulatory role in phagocytosis (Vinet et al., 2008), as well as in phagosome maturation, by controlling the acquisition of the $\mathrm{v}$-ATPase and of cathepsin D (Vinet et al., 2009). Thus, exclusion of Syt V from the phagosome membrane by LPG abrogates recruitment of the v-ATPase and impedes phagosome acidification (Vinet et al., 2009). Targeting the phagosome fusion machinery thus represents an efficient way to create an intracellular niche favorable to the establishment of a pathogen.

Shedding or secretion of glycans as a virulence mechanism to modulate phagosome maturation has been described for other intracellular pathogens, including $M$. tuberculosis, Brucella abortus, and Legionella pneumophila. Similar to LPG, the cyclic $\beta$-1,2-glucans of $B$. abortus and the lipoarabinomannan of $M$. tuberculosis impair phagolysosomal biogenesis by disrupting host cell lipid microdomains (Arellano-Reynoso et al., 2005; Welin et al., 2008). In the case of L. pneumophila, transmissive forms shed LPS-containing membrane vesicles that inhibit phagosome fusion with degradative lysosomes (Fernandez-Moreira et al., 2006). The exact mode of action

\section{REFERENCES}

Aderem, A., and Underhill, D. M. (1999). Mechanisms of phagocytosis in macrophages. Annu. Rev. Immunol. 17, 593-623.

Alexander, J., and Vickerman, K. (1975). Fusion of host cell secondary lysosomes with the parasitophorous vacuoles of Leishmania mexicana-infected macrophages. J. Protozool. 22, 502-508.

Allen, L. A., and McCaffrey, R. L. (2007). To activate or not to activate: distinct strategies used by Helicobacter pylori and Francisella tularensis to modulate the NADPH oxidase and survive in human neutrophils. Immunol. Rev. 219, 103-117.

Allen, L. H., and Aderem, A. (1995). A role for MARCKS, the alpha isozyme of protein kinase $\mathrm{C}$ and myosin I in zymosan phagocytosis by macrophages. J. Exp. Med. 182, 829-840.

Alvar, J., Velez, I. D., Bern, C., Herrero, M., Desjeux, P., Cano, J., Jannin, J., and Den Boer, M. (2012). Leishmaniasis worldwide and global estimates of its incidence. PLOS ONE 7:e35671. doi: 10.1371/journal.pone.0035671

Antoine, J. C., Prina, E., Jouanne, C., and Bongrand, P. (1990). Parasitophorous vacuoles of Leishmania amazonensis-infected macrophages maintain an acidic pH. Infect. Immun. 58, 779-787.
Antoine, J. C., Prina, E., Lang, T., and Courret, N. (1998). The biogenesis and properties of the parasitophorous vacuoles that harbour Leishmania in murine macrophages. Trends Microbiol. 6, 392-401.

Arellano-Reynoso, B., Lapaque, N., Salcedo, S., Briones, G., Ciocchini, A. E., Ugalde, R., Moreno, E., Moriyon, I., and Gorvel, J. P. (2005). Cyclic beta-1, 2-glucan is a Brucella virulence factor required for intracellular survival. Nat. Immunol. 6, 618-625.

Berman, J. D., Dwyer, D. M., and Wyler, D. J. (1979). Multiplication of Leishmania in human macrophages in vitro. Infect. Immun. 26 375-379.

Bokoch, G. M., and Diebold, B. A. (2002). Current molecular models for NADPH oxidase regulation by Rac GTPase. Blood 100, 2692-2696.

Brown, G. E., Stewart, M. Q., Liu, H., Ha, V. L., and Yaffe, M. B. (2003). A novel assay system implicates PtdIns(3, 4)P(2), PtdIns(3)P, and PKC delta in intracellular production of reactive oxygen species by the NADPH oxidase. Mol. Cell $11,35-47$.

Brumell, J. H., and Grinstein, S. (2004). Salmonella redirects phagosomal maturation. Curr. Opin. Microbiol. 7, 78-84.

Chang, K. P., and Dwyer, D. M. (1976). Multiplication of a human parasite (Leishmania donovani) in phagolysosomes of hamster

is not known. Whether these various bacterial glycans act by disabling the phagosome membrane fusion machinery remains to be further explored.

\section{CONCLUDING REMARK}

Similar to other intracellular pathogens, Leishmania promastigotes block the phagosome maturation process and create an environment which may be propitious to promastigote-to-amastigote differentiation. The surface glycolipid LPG plays a central role in this process. Further studies will be required to determine whether other Leishmania molecules are involved in the phagosome remodeling induced by promastigotes. Defining the functional properties of the promastigote-harboring vacuoles may also provide new insights into our understanding of the biology of Leishmania parasites, as well as the biology of phagolysosome biogenesis.

\section{ACKNOWLEDGMENTS}

Albert Descoteaux acknowledges financial support from the Canadian Institutes of Health Research (grant MOP-12933) and from the Canada Research Chairs program. Neda Moradin was partly supported by the Fondation Armand-Frappier.

macrophages in vitro. Science 193 678-680.

Chang, K. P., and Dwyer, D. M (1978). Leishmania donovani. Hamster macrophage interactions in vitro: cell entry, intracellular survival, and multiplication of amastigotes. J. Exp. Med. 147, 515-530.

Courret, N., Frehel, C., Gouhier, N., Pouchelet, M., Prina, E., Roux, P., and Antoine, J. C. (2002). Biogenesis of Leishmaniaharbouring parasitophorous vacuoles following phagocytosis of the metacyclic promastigote or amastigote stages of the parasites. J. Cell. Sci. 115, 2303-2316.

Dermine, J. F., Duclos, S., Garin, J., St-Louis, F., Rea, S., Parton, R. G., and Desjardins, M. (2001). Flotillin-1-enriched lipid raft domains accumulate on maturing phagosomes. J. Biol. Chem. 276, 18507-18512.

Dermine, J. F., Goyette, G., Houde, M., Turco, S. J., and Desjardins, M. (2005). Leishmania donovani lipophosphoglycan disrupts phagosome microdomains in $\mathrm{J774}$ macrophages. Cell. Microbiol. 7, 1263-1270.

Dermine, J. F., Scianimanico, S., Privé, C., Descoteaux, A., and Desjardins, M. (2000). Leishmania promastigotes require lipophosphoglycan to actively modulate the fusion properties of phagosomes at an early step of phagocytosis. Cell. Microbiol. 2, 115-126.
Descoteaux, A., Luo, Y., Turco, S. J., and Beverley, S. M. (1995). A specialized pathway affecting virulence glycoconjugates of Leishmania. Science 269, 1869-1872.

Descoteaux, A., and Turco, S. J. (1999). Glycoconjugates in Leishmania infectivity. Biochim. Biophys. Acta 1455, 341-352.

Desjardins, M., and Descoteaux, A. (1997). Inhibition of phagolysosomal biogenesis by the Leishmania lipophosphoglycan. J. Exp. Med. 185, 2061-2068.

Easterbrook, M. D., Levy, M. H., Gomez, A. M., Turco, S. J., Epand, R. M., and Rosenthal, K. L. (1995). Inhibition of HIV-1-induced syncytia formation and infectivity by lipophosphoglycan from Leishmania. J. Acquir. Immune Defic. Syndr. Hum. Retrovirol. 10, 496-505.

Fernandez-Moreira, E., Helbig, J. H., and Swanson, M. S. (2006) Membrane vesicles shed by Legionella pneumophila inhibit fusion of phagosomes with lysosomes. Infect. Immun. 74, 3285-3295.

Flannagan, R. S., Cosio, G., and Grinstein, S. (2009). Antimicrobial mechanisms of phagocytes and bacterial evasion strategies. Nat. Rev. Microbiol. 7, 355-366.

Forestier, C. L., Machu, C., Loussert, C., Pescher, P., and Spath, G. F. (2011). Imaging host cell-Leishmania interaction dynamics implicates parasite motility, lysosome recruitment, and 
host cell wounding in the infection process. Cell Host Microbe 9, 319-330.

Gagnon, E., Duclos, S., Rondeau, C., Chevet, E., Cameron, P. H., Steele-Mortimer, O., Paiement, J., Bergeron, J. J., and Desjardins, M. (2002). Endoplasmic reticulum-mediated phagocytosis is a mechanism of entry into macrophages. Cell 110, 119-131.

Gaur, U., Showalter, M., Hickerson, S., Dalvi, R., Turco, S. J., Wilson, M. E., and Beverley, S. M. (2009). Leishmania donovani lacking the Golgi GDP-Man transporter LPG2 exhibit attenuated virulence in mammalian hosts. Exp. Parasitol. 122, 182-191.

Gil, C., Soler-Jover, A., Blasi, J., and Aguilera, J. (2005). Synaptic proteins and SNARE complexes are localized in lipid rafts from rat brain synaptosomes. Biochem. Biophys. Res. Commun. 329, 117-124.

Giorgione, J. R., Turco, S. J., and Epand, R. M. (1996). Transbilayer inhibition of protein kinase C by the lipophosphoglycan from Leishmania donovani. Proc. Natl. Acad. Sci. U.S.A. 93, 11634-11639.

Harada, T., Miyake, M., and Imai, Y. (2007). Evasion of Legionella pneumophila from the bactericidal system by reactive oxygen species (ROS) in macrophages. Microbiol. Immunol. 51, 1161-1170.

Holm, A., Tejle, K., Gunnarsson, T., Magnusson, K. E., Descoteaux, A., and Rasmusson, B. (2003). Role of protein kinase $\mathrm{C}$ alpha for uptake of unopsonized prey and phagosomal maturation in macrophages. Biochem. Biophys. Res. Commun. 302, 653-658.

Holm, A., Tejle, K., Magnusson, K. E., Descoteaux, A., and Rasmusson, B. (2001). Leishmania donovani lipophosphoglycan causes periphagosomal actin accumulation: correlation with impaired translocation of PKCalpha and defective phagosome maturation. Cell. Microbiol. 3, 439-447.

Kay, J. G., Murray, R. Z., Pagan, J. K., and Stow, J. L. (2006). Cytokine secretion via cholesterolrich lipid raft-associated SNAREs at the phagocytic cup. J. Biol. Chem. 281, 11949-11954.

Lambertz, U., Silverman, J. M., Nandan, D., McMaster, W. R., Clos, J., Foster, L. J., and Reiner, N. E. (2012). Secreted virulence factors and immune evasion in visceral leishmaniasis. J. Leukoc. Biol. 91, 887-899.

Lerm, M., Holm, A., Seiron, A., Sarndahl, E., Magnusson, K. E., and
Rasmusson, B. (2006). Leishmania donovani requires functional $\mathrm{Cdc} 42$ and Racl to prevent phagosomal maturation. Infect. Immun. 74 2613-2618.

Lewis, D. H., and Peters, W. (1977) The resistance of intracellular Leishmania parasites to digestion by lysosomal enzymes. Ann. Trop. Med. Parasitol. 71, 295-312.

Lodge, R., and Descoteaux, A. (2005a). Leishmania donovani promastigotes induce periphagosomal F-actin accumulation through retention of the GTPase Cdc42. Cell. Microbiol. 7, 1647-1658.

Lodge, R., and Descoteaux, A. (2005b). Modulation of phagolysosome biogenesis by the lipophosphoglycan of Leishmania. Clin. Immunol. 114 256-265.

Lodge, R., and Descoteaux, A. (2006). Phagocytosis of Leishmania donovani amastigotes is Racl dependent and occurs in the absence of NADPH oxidase activation. Eur J. Immunol. 36, 2735-2744.

Lodge, R., Diallo, T. O., and Descoteaux, A. (2006). Leishmanio donovani lipophosphoglycan blocks $\mathrm{NADPH}$ oxidase assembly at the phagosome membrane. Cell. Microbiol. 8, 1922-1931.

Mantegazza, A. R., Savina, A., Vermeulen, M., Perez, L., Geffner J., Hermine, O., Rosenzweig, S. D., Faure, F., and Amigorena, S. (2008). NADPH oxidase controls phagosomal $\mathrm{pH}$ and antigen crosspresentation in human dendritic cells. Blood 112, 4712-4722.

Marshansky, V., and Futai, M. (2008). The V-type H+-ATPase in vesicular trafficking: targeting, regulation and function. Curr. Opin. Cell Biol. 20, 415-426.

McConville, M. J., and Blackwell, J. M. (1991). Developmental changes in the glycosylated phosphatidylinositols of Leishmania donovani. Characterization of the promastigote and amastigote glycolipids. J. Biol. Chem. 266, 15170-15179.

McConville, M. J., Thomas-Oates, J. E., Ferguson, M. A., and Homans, S. W. (1990). Structure of the lipophosphoglycan from Leishmania major. J. Biol. Chem. 265, 19611-19623.

Mukkada, A. J., Meade, J. C., Glaser, T. A., and Bonventre, P. F. (1985). Enhanced metabolism of Leishmania donovani amastigotes at acid $\mathrm{pH}$ : an adaptation for intracellular growth. Science 229, 1099-1101.

Ndjamen, B., Kang, B. H., Hatsuzawa, K., and Kima, P. E. (2010). Leishmania parasitophorous vacuoles interact continuously with the host cell's endoplasmic reticulum; parasitophorous vacuoles are hybrid compartments. Cell. Microbiol. 12 1480-1494.

Ng Yan Hing, J. D., Desjardins, M., and Descoteaux, A. (2004) Proteomic analysis reveals a role for protein kinase $\mathrm{C}$-alpha in phagosome maturation. Biochem. Biophys. Res. Commun. 319, 810-816.

Peters, N. C., and Sacks, D. L. (2009) The impact of vector-mediated neutrophil recruitment on cutaneous leishmaniasis. Cell. Microbiol. 11, 1290-1296.

Pham, N. K., Mouriz, J., and Kima, P. E. (2005). Leishmania pifano amastigotes avoid macrophage production of superoxide by inducing heme degradation. Infect. Immun 73, 8322-8333.

Real, F., Pouchelet, M., and Rabinovitch, M. (2008). Leishmania (L.) amazonensis: fusion between parasitophorous vacuoles in infected bone-marrow derived mouse macrophages. Exp. Parasitol. 119, 15-23.

Rodriguez, N. E., Gaur Dixit, U. Allen, L. A., and Wilson, M. E. (2011). Stage-specific pathways of Leishmania infantum chagasi entry and phagosome maturation in macrophages. PLoS ONE 6:e19000. doi: 10.1371/journal.pone.0019000

Rosenzweig, D., Smith, D., Opperdoes, F., Stern, S., Olafson, R. W., and Zilberstein, D. (2008). Retooling Leishmania metabolism: from sand fly gut to human macrophage. FASEB J. 22, 590-602.

Rybicka, J. M., Balce, D. R., Chaudhuri, S., Allan, E. R., and Yates, R. M. (2012). Phagosomal proteolysis in dendritic cells is modulated by NADPH oxidase in a $\mathrm{pH}$-independent manner. EMBO J. 31, 932-944.

Savina, A., Peres, A., Cebrian, I. Carmo, N., Moita, C., Hacohen, N., Moita, L. F., and Amigorena, S. (2009). The small GTPase Rac2 controls phagosomal alkalinization and antigen crosspresentation selectively in $\mathrm{CD} 8(+)$ dendritic cells. Immunity 30 544-555.

Scianimanico, S., Desrosiers, M. Dermine, J. F., Meresse, S. Descoteaux, A., and Desjardins, $\mathrm{M}$ (1999). Impaired recruitment of the small GTPase rab7 correlates with the inhibition of phagosome maturation by Leishmania donovan promastigotes. Cell. Microbiol. 1 , 19-32.

Shao, D., Segal, A. W., and Dekker, L. V. (2003). Lipid rafts determine efficiency of NADPH oxidase activation in neutrophils. FEBS Lett. 550, 101-106.

Silverman, J. M., Clos, J., De'oliveira, C. C., Shirvani, O., Fang, Y., Wang, C., Foster, L. J., and Reiner, N. E (2010). An exosome-based secretion pathway is responsible for protein export from Leishmania and communication with macrophages. J. Cell Sci. 123, 842-852.

Silverman, J. M., and Reiner, N. E. (2011). Exosomes and other microvesicles in infection biology: organelles with unanticipated phenotypes. Cell. Microbiol. 13, $1-9$.

Silverman, J. M., and Reiner, N. E. (2012). Leishmania exosomes deliver preemptive strikes to create an environment permissive for early infection. Front. Cell. Inf. Microbio. 1:8. doi: 10.3389/fcimb.2011.00026

Simons, K., and Vaz, W. L. (2004). Model systems, lipid rafts, and cell membranes. Annu. Rev. Biophys. Biomol. Struct. 33, 269-295.

Späth, G. F., Garraway, L. A., Turco, S. J., and Beverley, S. M. (2003). The role(s) of lipophosphoglycan (LPG) in the establishment of Leishmania major infections in mammalian hosts. Proc. Natl. Acad. Sci. U.S.A. 100, 9536-9541.

Thomas, J. R., McConville, M. J. Thomas-Oates, J. E., Homans, S. W., Ferguson, M. A., Gorin, P. A., Greis, K. D., and Turco, S. J. (1992) Refined structure of the lipophosphoglycan of Leishmania donovani. J. Biol. Chem. 267, 6829-6833.

Tolson, D. L., Turco, S. J., and Pearson, T. W. (1990). Expression of a repeating phosphorylated disaccharide lipophosphoglycan epitope on the surface of macrophages infected with Leishmania donovani. Infect. Immun. 58, 3500-3507.

Turco, S. J., and Sacks, D. L. (1991). Expression of a stage-specific lipophosphoglycan in Leishmania major amastigotes. Mol. Biochem. Parasitol. 45, 91-99.

Vazquez-Torres, A., and Fang, F. C. (2001). Salmonella evasion of the NADPH phagocyte oxidase. Microbes Infect. 3, 1313-1320.

Vilhardt, F., and Van Deurs, B. (2004). The phagocyte NADPH oxidase depends on cholesterolenriched membrane microdomains for assembly. EMBO J. 23, 739-748.

Vinet, A. F., Fukuda, M., and Descoteaux, A. (2008). The exocytosis regulator synaptotagmin $\mathrm{V}$ controls phagocytosis in macrophages. J. Immunol. 181, 5289-5295. 
Vinet, A. F., Fukuda, M., Turco, S. J., and Descoteaux, A. (2009). The Leishmania donovani lipophosphoglycan excludes the vesicular proton-ATPase from phagosomes by impairing the recruitment of synaptotagmin V. PLoS Pathog. 5:e1000628. doi: 10.1371/journal.ppat.1000628

Welin, A., Winberg, M. E., Abdalla, H., Sarndahl, E., Rasmusson, B., Stendahl, O., and Lerm, M. (2008). Incorporation of Mycobacterium tuberculosis lipoarabinomannan into macrophage membrane rafts is a prerequisite for the phagosomal maturation block. Infect. Immun. 76, 2882-2887.

Winberg, M. E., Holm, A., Sarndahl, E., Vinet, A. F., Descoteaux, A. Magnusson, K. E., Rasmusson, B., and Lerm, M. (2009). Leishmania donovani lipophosphoglycan inhibits phagosomal maturation via action on membrane rafts. Microbes Infect. 11, 215-222.

Wong, D., Bach, H., Sun, J., Hmama, Z., and Av-Gay, Y. (2011). Mycobacterium tuberculosis protein tyrosine phosphatase (PtpA) excludes host vacuolar-H+-ATPase to inhibit phagosome acidification.
Proc. Natl. Acad. Sci. U.S.A.108, 19371-19376.

Zilberstein, D., and Shapira, M. (1994).

The role of $\mathrm{pH}$ and temperature in the development of Leishmania parasites. Annu. Rev. Microbiol. 48, 449-470.

Conflict of Interest Statement: The authors declare that the research was conducted in the absence of any commercial or financial relationships that could be construed as a potential conflict of interest.

Received: 05 July 2012; paper pending published: 09 August 2012; accepted: 04
September 2012; published online: 19 September 2012

Citation: Moradin N and Descoteaux A (2012) Leishmania promastigotes: building a safe niche within macrophages. Front. Cell. Inf. Microbio. 2:121. doi: 10.3389/fcimb.2012.00121

Copyright (c) 2012 Moradin and Descoteaux. This is an open-access article distributed under the terms of the Creative Commons Attribution License, which permits use, distribution and reproduction in other forums, provided the original authors and source are credited and subject to any copyright notices concerning any third-party graphics etc. 